\title{
Target Localization in NLOS Environments Using RSS and TOA Measurements
}

\author{
Slavisa Tomic ${ }^{(\mathbb{0}}$, Marko Beko, Milan Tuba, and Victor M. Franco Correia
}

\begin{abstract}
This letter addresses the problem of target localization in adverse non-line-of-sight environments. By utilizing integrated received signal strength and time of arrival measurements, a novel alternating algorithm is proposed. The new algorithm is derived by converting the original nonconvex problem into a generalized trust region sub-problem framework, which can be solved exactly by just a bisection procedure. Therefore, the proposed algorithm is very light in terms of computational cost, and its excellent estimation accuracy is validated through computer simulations.
\end{abstract}

Index Terms-Localization in non-line-of-sight (NLOS), received signal strength (RSS), time of arrival (TOA), generalized trust region sub-problem (GTRS).

\section{INTRODUCTION}

$\mathbf{R}$ EGARDLESS of recent growing interest in indoor localization, present technologies have not converged towards a uniquely accepted solution. Hence, the topic still attracts wide research interest, owing to its constantly increasing application potential, as well as ample availability of smart-phones and advances in their embedded hardware. Besides, most people simply spend a large amount of time indoors (home, office, school, shopping malls, etc.) [1]-[4].

Much work has been done in the research society regarding target localization by means of existing terrestrial technologies recently [1]-[7]. Hybrid algorithms based on received signal strength (RSS) and angle of arrival were studied in [1] and [2], but only localization in line-of-sight (LOS) conditions was considered. In [3]-[5] and [7], hybrid algorithms based on RSS and time of arrival (TOA) were presented. Zhang et al. [3] and Coluccia and Fascista [4] studied the range estimation problem based on these two quantities, whereas the attention in [5] and [7] was on target

Manuscript received April 20, 2018; revised June 21, 2018; accepted July 18, 2018. Date of publication July 23, 2018; date of current version December 14, 2018. This work was supported in part by the Fundação para a Ciência e a Tecnologia under Project UID/EEA/00066/2013 and Project foRESTER PCIF/SSI/0102/2017, and in part by the Program Investigador FCT under Grant IF/00325/2015. The work of M. Tuba was supported by the Ministry of Education, Science and Technological Development of Republic of Serbia under Grant III-44006. The associate editor coordinating the review of this paper and approving it for publication was W. Hamouda. (Corresponding author: Slavisa Tomic.)

S. Tomic is with COPELABS, Universidade Lusófona de Humanidades e Tecnologias, 1749-024 Lisbon, Portugal, also with ISR/IST, LARSyS, Universidade de Lisboa, 1049-001 Lisbon, Portugal (e-mail: slavisa.tomic@ulusofona.pt).

M. Beko is with COPELABS, Universidade Lusófona de Humanidades e Tecnologias, 1749-024 Lisbon, Portugal, and also with CTS/UNINOVA, Campus da FCT/UNL, 2829-516 Caparica, Portugal (e-mail mbeko@uninova.pt).

M. Tuba is with the Department of Technical Sciences State, University of Novi Pazar, 36300 Novi Pazar, Serbia (e-mail: tuba@ieee.org).

V. M. F. Correia is with IDMEC Instituto Superior Técnico, Universidade de Lisboa, 1049-001 Lisbon, Portugal, and also with the Departamento de Engenharia Marítima, Escola Superior Náutica Infante D. Henrique, 2770-058 Paço de Arcos, Portugal (e-mail: victorfranco@enautica.pt).

Digital Object Identifier 10.1109/LWC.2018.2858248 localization problem. In the latter works, the authors proposed a weighted least squares (WLS) estimator for target localization in LOS/non-line of-sight (NLOS) environments and a robust least-squares multilateration technique to mitigate the negative effect of outliers, respectively.

This letter was inspired by an interesting discussion presented Coluccia and Fascista [4], where introduced a concept of a critical distance, below and above which one should make use of RSS-only and TOA-only measurements, respectively, and the combined measurements in the proximity of the critical distance to achieve advantage. Even though they presented some convincing arguments and results that justify their reasoning, the main focus in [4] was on range estimation problem in LOS environments and not the localization one. Contrary to [4], in this letter, we address the target localization problem in NLOS environments. The main objective of this letter is to investigate whether the hybrid approach is beneficial in terms of localization accuracy in NLOS environments over the traditional ones. Hence, by applying a squared range (SR) and WLS criterion to convert the originally nonconvex problem into a generalized trust region sub-problem (GTRS), we propose a novel alternating algorithm, called "SR-WLS". The SR-WLS can be solved exactly by means of bisection.

\section{Problem Formulation}

Let us denote by $\boldsymbol{x}$ and $\boldsymbol{a}_{i}$ the unknown location of the target and the known location of the $i$-th anchor $(i=$ $1, \ldots, N)$ in a $k$-dimensional plane $(k=2$ or 3$)$, respectively. Suppose that the target emits a signal to the anchors, which can then extract the RSS and the TOA information from the received signal. These two measurements can be modeled [3], [4] as

$$
\begin{aligned}
P_{i} & =P_{0}-b_{i}-10 \gamma \log _{10} \frac{\left\|\boldsymbol{x}-\boldsymbol{a}_{i}\right\|}{d_{0}}+n_{i}, \\
d_{i} & =\left\|\boldsymbol{x}-\boldsymbol{a}_{i}\right\|+\beta_{i}+m_{i},
\end{aligned}
$$

where $P_{0}$ is the RSS $(\mathrm{dBm})$ at a reference distance $d_{0}(\| \boldsymbol{x}-$ $\left.\boldsymbol{a}_{i} \| \geq d_{0}\right), b_{i}(\mathrm{~dB})$ and $\beta_{i}(\mathrm{~m})$ are the (positive) NLOS biases, $\gamma$ is the path loss exponent, $n_{i}$ is the log-normal shadowing term $(\mathrm{dB})$ modeled as $n_{i} \sim \mathcal{N}\left(0, \sigma_{n_{i}}^{2}\right)$, and $m_{i} \sim \mathcal{N}\left(0, \sigma_{m_{i}}^{2}\right)$ is the measurement noise $(\mathrm{m})$.

Based on (1), the joint RSS-TOA maximum likelihood (ML) estimator of $\boldsymbol{x}, b_{i}$ and $\beta_{i}$ is defined as

$$
\begin{aligned}
& \left\{\widehat{\boldsymbol{x}}, \widehat{b}_{i}, \widehat{\beta}_{i}\right\}=\underset{\boldsymbol{x}, b_{i}, \beta_{i}}{\arg \min } \sum_{i=1}^{N} \\
& \quad \frac{\left(P_{i}-P_{0}+b_{i}+10 \gamma \log _{10} \frac{\left\|x-a_{i}\right\|}{d_{0}}\right)^{2} \sigma_{m_{i}}^{2}+\left(d_{i}-\left\|x-a_{i}\right\|-\beta_{i}\right)^{2} \sigma_{n_{i}}^{2}}{\sigma_{n_{i}}^{2} \sigma_{m_{i}}^{2}}
\end{aligned}
$$

The above estimator is the exact ML one if the RSS and TOA observations are taken from independent sources [4]. 
Nonetheless, experiments in [3] and [9] show that the observations extracted from the same signal are weakly correlated; thus, the assumption of uncorrelated measurements is not unreasonable.

The problem in (2) is highly non-convex and has no closedform solution. Moreover, in practice, we can not distinguish between LOS/NLOS links; thus, (2) is also under-determined, since the number of unknowns $(2 N+k)$ is greater than the number of observations $(2 N)$. Therefore, (2) can not be tackled directly, and we develop a different estimator in Section III.

\section{The Proposed Hybrid Estimator}

In this section, a detailed derivation of the proposed hybrid algorithm is described. First, we approximate (1) by

$$
\begin{aligned}
P_{i} & =P_{0}-b-10 \gamma \log _{10} \frac{\left\|\boldsymbol{x}-\boldsymbol{a}_{i}\right\|}{d_{0}}+n_{i}, \\
d_{i} & =\left\|\boldsymbol{x}-\boldsymbol{a}_{i}\right\|+\beta+m_{i},
\end{aligned}
$$

i.e., we convert the originally under-determined problem into a determined one (for $2 N \geq k+2$ ). In other words, we approximate the $N$ NLOS biases in each type of measurement by a single (mean) one, called a balancing parameter. The price we pay for such an approximation is only partial mitigation of the NLOS bias. However, on the other hand, we keep the balancing parameters, $b$ and $\beta$, as optimization variables to be estimated together with the target location, which leaves us with somewhat control over the problem at hand (e.g., in the two extreme cases: all LOS/NLOS links).

We can rearrange $(3 a)$ as

$$
10^{\frac{P_{0}-P_{i}-b+n_{i}}{10 \gamma}}=\frac{\left\|\boldsymbol{x}-\boldsymbol{a}_{i}\right\|}{d_{0}},
$$

which, by applying the first order Taylor series of the form $\mathrm{e}^{t} \approx 1+t$ for small $t$, can be approximated by

$$
\rho+\epsilon_{i} \approx \xi_{i}\left\|\boldsymbol{x}-\boldsymbol{a}_{i}\right\|
$$

with $\rho=d_{0} 10^{\frac{P_{0}-b}{10 \gamma}}, \xi_{i}=10^{\frac{P_{i}}{10 \gamma}}, \epsilon_{i} \sim \mathcal{N}\left(0,\left(\rho \frac{\ln (10)}{10 \gamma} \sigma_{n_{i}}\right)^{2}\right)$.

Rearranging and squaring (4) and (3b) yields

$$
\begin{aligned}
\rho^{2} & \approx \xi_{i}^{2}\left\|\boldsymbol{x}-\boldsymbol{a}_{i}\right\|^{2}-2 \epsilon_{i} \xi_{i}\left\|\boldsymbol{x}-\boldsymbol{a}_{i}\right\|+\epsilon_{i}^{2}, \\
\left(d_{i}-\beta\right)^{2} & =\left\|\boldsymbol{x}-\boldsymbol{a}_{i}\right\|^{2}-2 m_{i}\left\|\boldsymbol{x}-\boldsymbol{a}_{i}\right\|+m_{i}^{2},
\end{aligned}
$$

respectively, from which we can write ${ }^{1}$

$$
\begin{gathered}
\frac{\xi_{i}^{2}\left\|\boldsymbol{x}-\boldsymbol{a}_{i}\right\|^{2}-\rho^{2}}{2 \xi_{i}\left\|\boldsymbol{x}-\boldsymbol{a}_{i}\right\|} \approx \epsilon_{i}-\frac{\epsilon_{i}^{2}}{2 \xi_{i}\left\|\boldsymbol{x}-\boldsymbol{a}_{i}\right\|}, \\
\frac{\left\|\boldsymbol{x}-\boldsymbol{a}_{i}\right\|^{2}-\left(d_{i}-\beta\right)^{2}}{2\left\|\boldsymbol{x}-\boldsymbol{a}_{i}\right\|}=m_{i}-\frac{m_{i}^{2}}{\left\|\boldsymbol{x}-\boldsymbol{a}_{i}\right\|} .
\end{gathered}
$$

Define weights, $w_{R i}=1-\widehat{d}_{i} / \sum_{i=1}^{N} \widehat{d}_{i}$ and $w_{T i}=1-$ $\ddot{d}_{i} / \sum_{i=1}^{N} \ddot{d}_{i}$, where $\widehat{d}_{i}=d_{0} 10^{\frac{P_{0}-P_{i}-b}{10 \gamma}}$ represents the ML estimate of the distance from (1a) and $\ddot{d}_{i}=d_{i}-\beta$, so that

\footnotetext{
${ }^{1}$ Notice that by squaring (4) and (3b), $\epsilon_{i}$ and $m_{i}$ are not Gaussian anymore Nevertheless, for sufficiently low noise power, we can disregard the secondorder noise terms and make use of the first-order noise terms only.
}

more relevance is given to nearby links. By applying a WLS criterion to (6), estimates of $x, b$ and $\beta$ can be obtained as

$$
\begin{aligned}
\underset{\boldsymbol{x}, b, \beta}{\operatorname{minimize}} & \sum_{i=1}^{N} w_{R i}\left(\frac{\xi_{i}^{2}\left\|\boldsymbol{x}-\boldsymbol{a}_{i}\right\|^{2}-\rho^{2}}{2 \xi_{i}\left\|\boldsymbol{x}-\boldsymbol{a}_{i}\right\|}\right)^{2} \\
& +\sum_{i=1}^{N} w_{T i}\left(\frac{\left\|\boldsymbol{x}-\boldsymbol{a}_{i}\right\|^{2}-\ddot{d}_{i}^{2}}{2\left\|\boldsymbol{x}-\boldsymbol{a}_{i}\right\|}\right)^{2} .
\end{aligned}
$$

The problem in (7) is highly non-convex. Therefore, instead of tackling (7) directly, we substitute it by

$$
\begin{aligned}
\underset{\boldsymbol{x}, b, \beta}{\operatorname{minimize}} & \sum_{i=1}^{N} w_{R i}\left(\frac{\xi_{i}^{2}\left\|\boldsymbol{x}-\boldsymbol{a}_{i}\right\|^{2}-\rho^{2}}{2 \xi_{i} \widehat{d}_{i}}\right)^{2} \\
& +\sum_{i=1}^{N} w_{T i}\left(\frac{\left\|\boldsymbol{x}-\boldsymbol{a}_{i}\right\|^{2}-\ddot{d}_{i}^{2}}{2 \ddot{d}_{i}}\right)^{2} .
\end{aligned}
$$

Although there are no guarantees that by minimizing (8), one also minimizes (7), the latter problem is a tight approximation of the previous one for small noise. ${ }^{2}$

Observe that if $b$ and $\beta$ are known, (8) could be solved exactly by a bisection procedure. This motivates us to apply an alternating procedure [8] to estimate $x$, and $b$ and $\beta$. The proposed estimation procedure comprises the following steps.

1) Initialize $b$ and $\beta: \widehat{b}=0$ and $\widehat{\beta}=0$;

2) Define $\widehat{\rho}=\left.\rho\right|_{b=\widehat{b}}, \breve{d}_{i}=\left.\widehat{d}_{i}\right|_{b=\widehat{b}}$ and $\tilde{d}_{i}=\left.\ddot{d}_{i}\right|_{\beta=\widehat{\beta}}$ and expand the numerators [10] of (8) to get

$$
\underset{y=\left[\boldsymbol{x}^{T},\|\boldsymbol{x}\|^{2}\right]^{T}}{\operatorname{minimize}}\left\{\|\boldsymbol{W}(\boldsymbol{A} \boldsymbol{y}-\boldsymbol{p})\|^{2}: \boldsymbol{y}^{T} \boldsymbol{D} \boldsymbol{y}+2 \boldsymbol{g}^{T} \boldsymbol{y}=0\right\},
$$

where $\boldsymbol{W}=\operatorname{diag}\left(\left[\widetilde{\boldsymbol{w}}_{1}^{T}, \widetilde{\boldsymbol{w}}_{2}^{T}\right]\right), \widetilde{\boldsymbol{w}}_{1}=\left[\widetilde{w}_{1 i}\right]^{T}, \widetilde{w}_{1 i}=$ $\frac{\sqrt{w_{R i}}}{2 \xi_{i} \breve{d}_{i}}, \widetilde{\boldsymbol{w}}_{2}=\left[\widetilde{w}_{2 i}\right]^{T}, \widetilde{w}_{2 i}=\frac{\sqrt{w_{T i}}}{2 \tilde{d}_{i}}$,

$$
\begin{aligned}
& \boldsymbol{A}=\left[\begin{array}{cc}
\vdots & \vdots \\
2 \xi_{i}^{2} \boldsymbol{a}_{i}^{T} & -\xi_{i}^{2} \\
\vdots & \vdots \\
2 \boldsymbol{a}_{i}^{T} & -1 \\
\vdots & \vdots
\end{array}\right], \boldsymbol{p}=\left[\begin{array}{c}
\vdots \\
\xi_{i}^{2}\left\|\boldsymbol{a}_{i}\right\|^{2}-\widehat{\rho}^{2} \\
\vdots \\
\left\|\boldsymbol{a}_{i}\right\|^{2}-\tilde{d}_{i}^{2} \\
\vdots
\end{array}\right], \\
& \boldsymbol{D}=\left[\begin{array}{cc}
\boldsymbol{I}_{k} & \mathbf{0}_{k \times 1} \\
\mathbf{0}_{1 \times k} & 0
\end{array}\right], \boldsymbol{g}=\left[\begin{array}{c}
\mathbf{0}_{k \times 1} \\
-1 / 2
\end{array}\right]
\end{aligned}
$$

3) Exploit the estimate of $\boldsymbol{x}, \hat{\boldsymbol{x}}$, obtained from (9) to find an ML estimate of the balancing terms, ${ }^{3}$ i.e.,

$$
\begin{aligned}
\widehat{b} & =\frac{\sum_{i=1}^{N}\left(P_{0}-P_{i}-10 \gamma \log _{10} \frac{\left\|\hat{\boldsymbol{x}}-\boldsymbol{a}_{i}\right\|}{d_{0}}\right)}{N}, \\
\widehat{\beta} & =\frac{\sum_{i=1}^{N}\left(d_{i}-\left\|\hat{\boldsymbol{x}}-\boldsymbol{a}_{i}\right\|\right)}{N} ;
\end{aligned}
$$

\section{4) $\operatorname{Redo}^{4}$ Step 2).}

\footnotetext{
${ }^{2}$ The distance that best estimates $\left\|\boldsymbol{x}-\boldsymbol{a}_{i}\right\|$ in (3b) in the ML sense is $d_{i}-\beta=\ddot{d}_{i}$. The same approach was applied for the RSS part.

${ }^{3}$ Note that, if any of the two estimates was negative, we set it to zero, i.e., all the links are treated as LOS, since the NLOS bias is positive [6].

${ }^{4}$ One could iterate between the Steps 2)-4) until a certain criterion is met. In our simulations however, we found that two iterations are sufficient.
} 
Both the objective function and the constraint in (9) are quadratic; it falls into the category of GTRS [10], whose exact solution can be readily obtained by a bisection procedure.

\section{Performance Assessment}

This section presents a set of simulation results in order to validate the performance of the proposed algorithm. The considered algorithms were implemented in MATLAB, and the radio measurements were generated according to (1). All sensors were deployed randomly inside an area of $B \times B \mathrm{~m}^{2}$ in each Monte Carlo, $M_{c}$ run. Other simulation parameters were fixed as: $P_{0}=20 \mathrm{dBm}, \gamma=3, d_{0}=1 \mathrm{~m}$, and $M_{c}=$ 10000. Moreover, both NLOS biases (RSS and TOA) were drawn randomly from a uniform distribution on the interval $\left[0\right.$, bias $\left._{\max }\right](\mathrm{dB}, \mathrm{m})$, i.e., bias $_{i} \sim \mathcal{U}\left[0\right.$, bias $\left._{\max }\right], i=1, \ldots, N$ in each $M_{c}$ run. The performance metric is the root mean squared error (RMSE), RMSE $=\sqrt{\sum_{i=1}^{M_{c}} \frac{\left\|\boldsymbol{x}_{i}-\widehat{\boldsymbol{x}}_{i}\right\|}{M_{c}}}$, where $\widehat{\boldsymbol{x}}_{i}$ denotes the estimate of the true target location, $\boldsymbol{x}_{i}$, in the $i$-th $M_{c}$ run.

The new algorithm is compared to those described in [3], [4], and [7] labeled here as "NR", "JAH" and "WLS", respectively. Even though the NR and the JAH are originally designed to estimate the distance between the target and the $i$-th anchor, the application of the bisection principle is straightforward after these estimates are obtained. Furthermore, in order to test the hypothesis that the hybrid algorithm performs better than the traditional ones, the results of the proposed algorithm using exclusively RSS or TOA measurements, denoted here by "SR - WLS RSS" and "SR - WLS TOA", respectively, are included. Hence, in terms of computational complexity, all considered algorithms are linear with $N$, i.e., their worst case computational complexity is $\mathcal{O}(K N)$, with $K$ representing the maximum number of steps in the bisection procedure. In all simulations, $K=30$ is used. Apart from the fact that the new approach comprises two iterations to reach the final solution, the only other significant difference in the computational cost of the algorithms is that the JAH requires a computation of the principal branch of the Lambert W-function [4]. Finally, an exhaustive grid search for an idealistic case (perfectly known NLOS bias realizations and noise powers) is applied to obtain a solution of (2) and (7). This brute force method is applied across the whole region with $0.1 \mathrm{~m}$ step size and is used here as a lower bound on the performance of the proposed approach. Note however that such a bound is hardly attainable in practice (due to system parameters idealization and time execution).

Fig. 1 illustrates the RMSE (m) versus $N$ performance comparison. ${ }^{5}$ As expected, all algorithms better as $N$ increases. However, the proposed hybrid algorithm has clearly the best performance for all $N$. Fig. 1 shows that, in comparison with the hybrid algorithms, the proposed one benefits significantly more with the increase of $N$. This behavior of the existing algorithms is somewhat surprising and indicates that the NR and the JAH get saturated much faster than the new algorithm. Moreover, in comparison with the traditional algorithms, one can notice that the performance margin gradually reduces as $N$ increases. This is anticipated to some extent, since when $N$ is large, the information gathered within the network is sufficient to allow relatively high accuracy to all algorithms. It can also

${ }^{5}$ It is important to note that the WLS, NR and JAH algorithms require knowledge about $b_{i}, \beta_{i}$ and $\sigma_{n_{i}}, \sigma_{m_{i}}$. Therefore, in all figures presented here, the true values of these parameters were given to these algorithms.

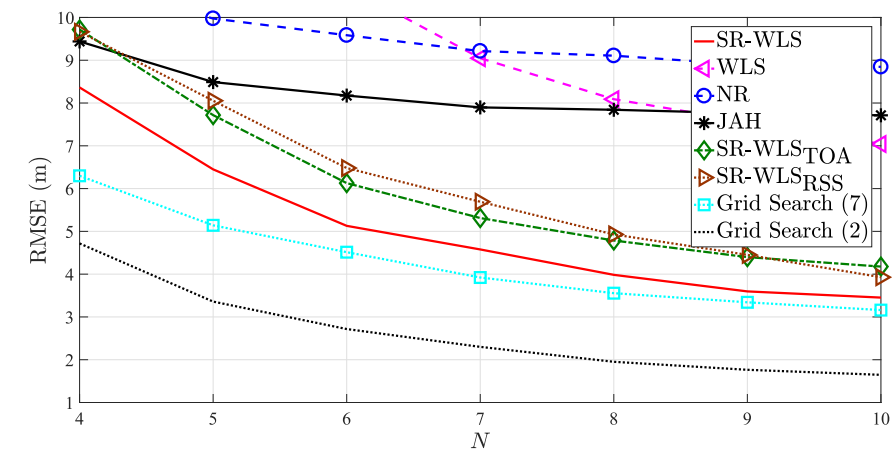

Fig. 1. RMSE versus $N$ comparison, when $N_{\text {nlos }}=N, \sigma_{n_{i}}=3 \mathrm{~dB}$, $\sigma_{m_{i}}=4 \mathrm{~m}$, biasmax $=6(\mathrm{~dB}, \mathrm{~m})$, bias $_{i} \sim \mathcal{U}[0$, biasmax $], B=30 \mathrm{~m}$.

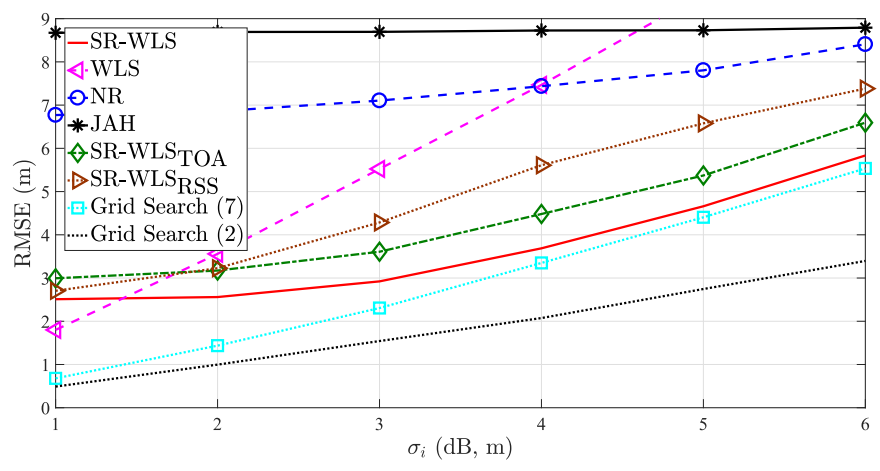

Fig. 2. RMSE versus $\sigma_{i}(\mathrm{~dB}, \mathrm{~m})$ comparison, when $N=9, N_{\text {nlos }}=9$, bias $_{\max }=6(\mathrm{~dB}, \mathrm{~m})$, bias $_{i} \sim \mathcal{U}\left[0\right.$, biasmax $_{\operatorname{man}}, B=30 \mathrm{~m}$.

be seen that the proposed algorithm practically achieves the lower bound set by the grid search of (7) for high $\mathrm{N}$, indicating that the applied relaxations are in the right direction.

Fig. 2 illustrates the RMSE (m) versus noise powers, $\sigma_{i}(\mathrm{~dB}, \mathrm{~m})$, performance comparison. This is an important scenario, since the new algorithm is an approximation of the ML one for small $\sigma_{i}$. Notice that, in favor of checking the influence of the noise power on the localization error, the values of the NLOS biases are fixed. Fig. 2 shows that all algorithms suffer impairments in performance accuracy as $\sigma_{i}$ grows, as anticipated. Nevertheless, in sharp contrast to the existing algorithms, the new one does not require to know $\sigma_{i}$ or $b_{i}, \beta_{i}$. Finally, it can also be seen that the proposed algorithm has the best performance for almost all considered span of $\sigma_{i}$.

Fig. 3 illustrates the RMSE $(\mathrm{m})$ versus $\operatorname{bias}_{\max }(\mathrm{dB}, \mathrm{m})$ performance comparison. Similarly as before, in favor of investigating the influence of the NLOS bias, the noise power is fixed. The figure exhibits relatively low deteriorations in accuracy of the proposed algorithm. This result is significant, since it corroborates our intuition to first treat all links as LOS and derive an alternating localization algorithm. Once again, the existing algorithms show stable performance because they are given the true values of the NLOS biases and noise powers.

Fig. 4 illustrates the RMSE (m) versus the number of NLOS links, $N_{\text {nlos }}$, performance comparison. The figure shows that all considered algorithms are practically robust to the relation of LOS/NLOS links. This was foreseen for the existing algorithms (using true values of the NLOS biases), and can be seen as a confirmation of the quality of the approximation steps taken in the derivation of the proposed algorithm. 


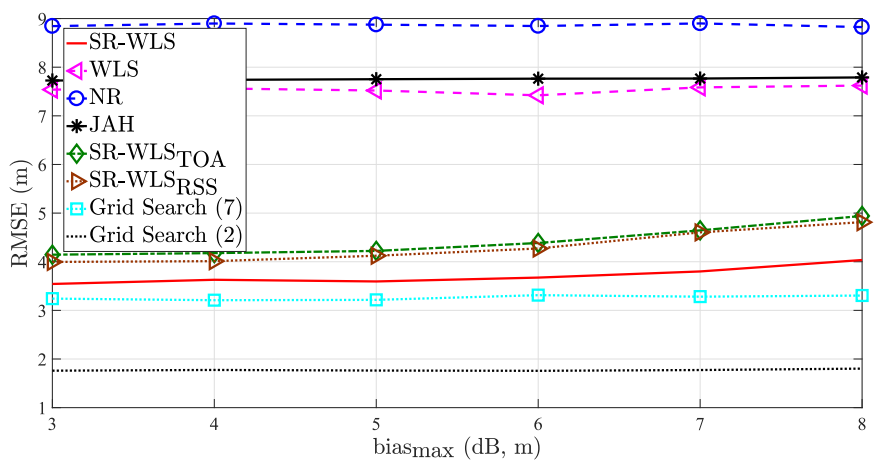

Fig. 3. RMSE versus bias $\max (\mathrm{dB}, \mathrm{m})$ comparison, when $N=9, N_{\text {nlos }}=9$, $\sigma_{n_{i}}=3 \mathrm{~dB}, \sigma_{m_{i}}=4 \mathrm{~m}, \operatorname{bias}_{i} \sim \mathcal{U}[0$, biasmax $], B=30 \mathrm{~m}$.

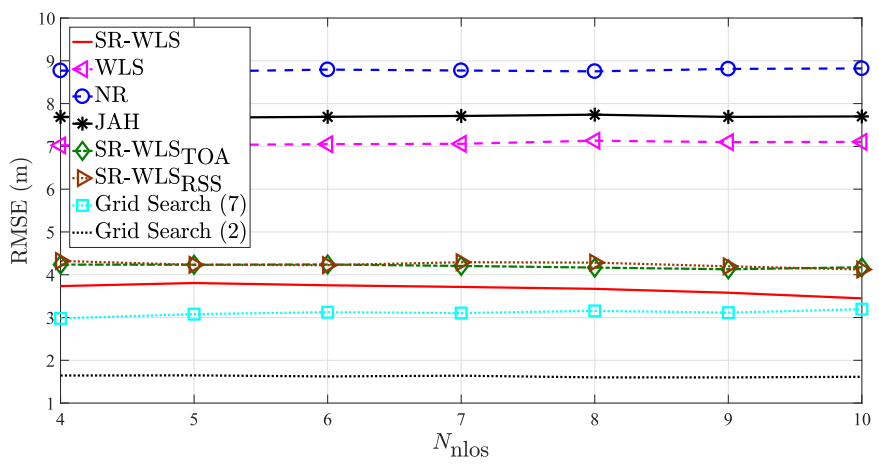

Fig. 4. RMSE versus $N_{\text {nlos }}$ comparison, when $N=10, \sigma_{n_{i}}=3 \mathrm{~dB}$, $\sigma_{m_{i}}=4 \mathrm{~m}, \operatorname{bias}_{\max }=6(\mathrm{~dB}, \mathrm{~m}), \operatorname{bias}_{i} \sim \mathcal{U}\left[0, \operatorname{bias}_{\max }\right], B=30 \mathrm{~m}$.

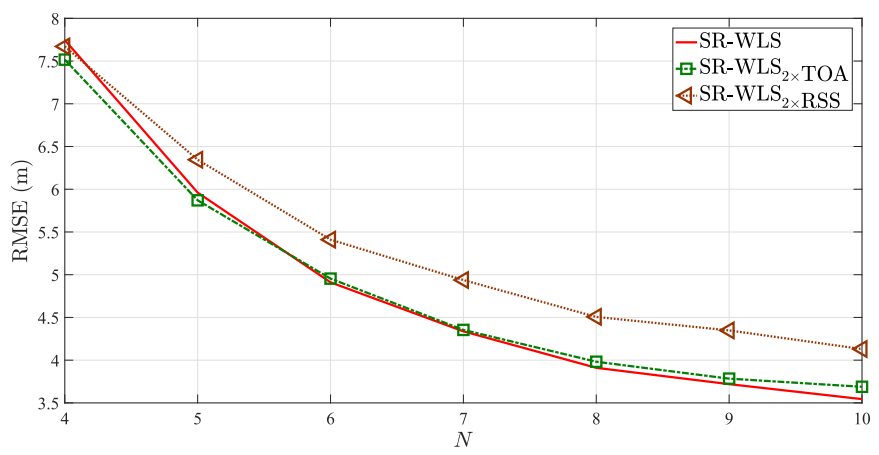

Fig. 5. RMSE versus $N$ comparison, when $N_{\text {nlos }}=N, \sigma_{n_{i}}=4 \mathrm{~dB}$, $\sigma_{m_{i}}=4 \mathrm{~m}$, bias $_{\max }=6(\mathrm{~dB}, \mathrm{~m})$, bias $_{i} \sim \mathcal{U}\left[0\right.$, biasmax $\left._{\max }\right], B=25 \mathrm{~m}$.

Lastly, it would be interesting to study the performance of the SR-WLS against itself, using a set of double RSS-only or TOA-only observations. Results for such a comparison are presented in Fig. 5, which shows that all algorithms have roughly the same performance. Although the comparison in terms of quantity of the acquired information is fair, it is important to note that, in order to acquire the double measurements, $\mathrm{SR}-\mathrm{WLS}_{\mathrm{RSS}}$ and $\mathrm{SR}-\mathrm{WLS}_{\mathrm{TOA}}$ require two signal transmissions. This might affect the sensors' battery lives in long term, as well as the utilization efficiency of the radio spectrum (doubling the measurement time and increasing the risk of message collisions). On the other hand, the hybrid algorithms require a single transmission to acquire two measurements (RSS and TOA), at a cost of a somewhat increased complexity of the sensors in terms of hardware. Nevertheless, recent advances in micro electro-mechanical systems allow practically all modern devices to measure these two quantities [11], [12].

\section{CONClusion}

In this letter, the problem of target localization in adverse NLOS environments by using fused RSS and TOA measurements was addressed. By approximating the $N$ NLOS biases with a single (mean) one, a novel algorithm, based on merely a bisection procedure was proposed. The new algorithm is an alternating one, in which the values of the balancing terms were first fixed in order to obtain a location estimate. Afterwards, the values of these terms were updated and exploited to enhance the localization accuracy. In our simulations, we found that two iterations were sufficient to achieve good accuracy. The simulation results confirmed the effectiveness of such an approach, outperforming the existing hybrid algorithms. Furthermore, the new hybrid algorithm was tested against itself when employing RSS-only and TOA-only measurements. Similar with the findings presented in [4], our results showed that the hybrid estimator can indeed outperform the traditional ones even in NLOS environments.

\section{ACKNOWLEDGMENT}

The authors would like to express sincere gratitude to Prof. Angelo Coluccia and Eng. Alessio Fascista from University of Salento, Italy, for their help in implementing their algorithm, and a fruitful discussion about critical distance.

\section{REFERENCES}

[1] S. Tomic, M. Beko, and R. Dinis, "Distributed RSS-AoA based localization with unknown transmit powers," IEEE Wireless Commun. Lett., vol. 5, no. 4, pp. 392-395, Aug. 2016.

[2] S. Tomic, M. Beko, R. Dinis, and P. Montezuma, "A closed-form solution for RSS/AoA target localization by spherical coordinates conversion," IEEE Wireless Commun. Lett., vol. 5, no. 6, pp. 680-683, Dec. 2016.

[3] J. Zhang, L. Ding, Y. Wang, and L. Hu, "Measurement-based indoor NLOS TOA/RSS range error modelling," Electron. Lett., vol. 52, no. 2, pp. 165-167, Jan. 2016.

[4] A. Coluccia and A. Fascista, "On the hybrid TOA/RSS range estimation in wireless sensor networks," IEEE Trans. Wireless Commun., vol. 17, no. 1, pp. 361-371, Jan. 2018.

[5] A. Bahillo et al., "Hybrid RSS-RTT localization scheme for indoor wireless networks," EURASIP J. Adv. Signal Process., vol. 2010, pp. 1-12, Mar. 2010.

[6] S. Tomic, M. Beko, R. Dinis, and P. Montezuma, "A robust bisectionbased estimator for TOA-based target localization in NLOS environments," IEEE Commun. Lett., vol. 21, no. 11, pp. 2488-2491, Nov. 2017.

[7] S. Tiwari, D. Wang, M. Fattouche, and F. Ghannouchi, "A hybrid RSS/TOA method for 3D positioning in an indoor environment," ISRN Signal Process., vol. 2012, p. 9, Jan. 2012.

[8] S. Tomic, M. Beko, and R. Dinis, "RSS-based Localization in wireless sensor networks using convex relaxation: Noncooperative and cooperative schemes," IEEE Trans. Veh. Technol., vol. 64, no. 5, pp. 2037-2050, May 2015.

[9] D. Macii, A. Colombo, P. Pivato, and D. Fontanelli, "A data fusion technique for wireless ranging performance improvement," IEEE Trans. Instrum. Meas., vol. 62, no. 1, pp. 27-37, Jan. 2013.

[10] A. Beck, P. Stoica, and J. Li, "Exact and approximate solutions of source localization problems," IEEE Trans. Sign. Process., vol. 56, no. 5, pp. 1770-1778, May 2008.

[11] S. Tomic, M. Beko, and R. Dinis, "3-D target localization in wireless sensor network using RSS and AoA measurement," IEEE Trans. Veh. Technol., vol. 66, no. 4, pp. 3197-3210, Apr. 2017.

[12] N. Patwari et al., "Locating the nodes: Cooperative localization in wireless sensor networks," IEEE Signal Process. Mag., vol. 22, no. 4, pp. 54-69, Jul. 2005. 